\title{
A new family of Frobenius-Genocchi polynomials and its certain properties
}

\author{
Ugur Duran $^{a}$, Mehmet Acikgoz ${ }^{b}$ and Serkan Araci ${ }^{c, *}$ \\ ${ }^{a}$ Department of Basic Sciences of Engineering, \\ Faculty of Engineering and Natural Sciences, \\ Iskenderun Tecnical University, TR-31200 Hatay, Turkey \\ E-Mail: mtdrnugur@gmail.com \\ ${ }^{b}$ Department of Mathematics, Faculty of Arts and Science, \\ University of Gaziantep, TR-27310 Gaziantep, Turkey \\ E-Mail: acikgoz@gantep.edu.tr \\ ${ }^{c}$ Department of Economics, Faculty of Economics, \\ Administrative and Social Science, \\ Hasan Kalyoncu University, TR-27410 Gaziantep, Turkey \\ E-Mail: mtsrkn@hotmail.com \\ ${ }^{*}$ Corresponding Author
}

\begin{abstract}
Recently, Kim-Kim [13] have introduced polyexponential functions as an inverse to the polylogarithm functions, and constructed type 2 poly-Bernoulli polynomials. They have also introduced unipoly functions attached to each suitable arithmetic function as a universal concept. Inspired by their work, in this paper, we introduce a new class of the Frobenius-Genocchi polynomials. We derive the diverse formulas and identities covering some summation formulas, derivative formula and correlations with Bernoulli polynomials and numbers, Stirling numbers of the both kinds, degenerate Frobenius-Genocchi polynomials and degenerate Frobenius-Euler polynomials. Moreover, by using the unipoly function as following Kim-Kim's work in [13], we consider degenerate unipoly-Frobenius-Genocchi polynomials and investigate some formulas and relationships with Daehee numbers, degenerate Frobenius-Genocchi numbers and Stirling numbers of the first kind. Finally, we obtain an Gaussian integral representation of the Frobenius-Genocchi polynomials in terms of the 2-variable Hermite polynomials.
\end{abstract}

2010 Mathematics Subject Classification- 11B83, 11S80, 05A19.

Keywords and Phrases- Polyexponential function; Degenerate exponential function; Polyexponential function; Frobenius-Genocchi polynomials; Poly-Frobenius-Genocchi polynomials.

\section{INTRODUCTION}

The special polynomials that can be defined in a various ways such as by generating functions, by recurrence relations, by $p$-adic integrals, by the degenerate versions, etc. provide new means of analysis in the solution of a wide class of differential or partial differential equations often encountered in the field of physical problems.

Recently, using polyexponential function with unipoly function in [13] and its degenerate version in [16], several new extensions of some special polynomials such as Frobenius-Genocchi polynomials by Duran et al. [7], Bernoulli polynomials by Kim et al. [16, 17], Bernoulli polynomials of the second kind by Kim et al. [19], Euler polynomials by Lee et al. [22] and Genocchi polynomials by Qin [24] have been extensively investigated.

Throughout of the paper we make use of the following notations: $\mathbb{N}:=\{1,2,3, \cdots\}$ and $\mathbb{N}_{0}=\mathbb{N} \cup\{0\}$. Here, as usual, $\mathbb{Z}$ denotes the set of all integers, $\mathbb{R}$ denotes the set of all real numbers and $\mathbb{C}$ denotes the set of all complex numbers.

Let $(x)_{n, \lambda}$ be $\lambda$-falling factorial given by

$$
(x)_{0, \lambda}:=1, \quad(x)_{n, \lambda}=x(x-\lambda)(x-2 \lambda) \cdots(x-(n-1) \lambda),
$$


and then the degenerate exponential function $e_{\lambda}^{x}(t)$ is defined by

$$
e_{\lambda}^{x}(t):=(1+\lambda t)^{\frac{x}{\lambda}}=\sum_{n=0}^{\infty}(x)_{n, \lambda} \frac{t^{n}}{n !} \text { and } e_{\lambda}^{1}(t):=e_{\lambda}(t), \text { see }[15,16,17,19,20] .
$$

The degenerate Bernoulli $B_{n, \lambda}(x)$, the degenerate Euler $E_{n, \lambda}(x)$ and the degenerate Genocchi $G_{n, \lambda}(x)$ polynomials are defined by

$$
\sum_{n=0}^{\infty} B_{n, \lambda}(x) \frac{t^{n}}{n !}=\frac{t}{e_{\lambda}(t)-1} e_{\lambda}^{x}(t) \text { and } \sum_{n=0}^{\infty} E_{n, \lambda}(x) \frac{t^{n}}{n !}=\frac{2}{e_{\lambda}(t)+1} e_{\lambda}^{x}(t),
$$

and

$$
\sum_{n=0}^{\infty} G_{n, \lambda}(x) \frac{t^{n}}{n !}=\frac{2 t}{e_{\lambda}(t)+1} e_{\lambda}^{x}(t)
$$

see $[7,15,16,17,19,20,22]$.

It is obvious that

$$
\lim _{\lambda \rightarrow 0} B_{n, \lambda}(x):=B_{n}(x), \lim _{\lambda \rightarrow 0} E_{n, \lambda}(x):=E_{n}(x) \text { and } \lim _{\lambda \rightarrow 0} G_{n, \lambda}(x):=G_{n}(x)
$$

where $B_{n}(x), E_{n}(x)$ and $G_{n}(x)$ are the Bernoulli polynomials, Euler polynomials and Genocchi polynomials, respectively, $c f .[4,5,18,25]$.

Frobenius studied the polynomials $F_{n}(x \mid u)$ given by $(c f .[3,21])$

$$
\frac{1-u}{e^{t}-u} e^{x t}=\sum_{n=0}^{\infty} F_{n}(x \mid u) \frac{t^{n}}{n !} \quad\left(u \in \mathbb{C} \backslash\{1\} ; e^{t} \neq u\right) .
$$

Upon setting $u=-1$, it becomes

$$
F_{n}(x \mid-1)=E_{n}(x) .
$$

Owing to relationship with the Euler polynomials as well as their important properties, and in the honor of Frobenius, the aforementioned polynomials denoted by $F_{n}(x \mid u)$ are called the Frobenius-Euler polynomials, cf. $[3,21]$. by

Parallel to (1.5), Yaşar and Özarslan [27] introduced the Frobenius-Genocchi polynomials $G_{n}^{F}(x ; u)$ given

$$
\frac{(1-u) t}{e^{t}-u} e^{x t}=\sum_{n=0}^{\infty} G_{n}^{F}(x ; u) \frac{t^{n}}{n !}
$$

because of the following relation

$$
G_{n}^{F}(x ;-1)=G_{n}(x) .
$$

The case $x=0$ in (1.6), $G_{n}^{F}(0 ; u):=G_{n}^{F}(u)$ stands for the Frobenius-Genocchi numbers. Several recurrence relations and differential equations are also investigated in [27].

The Bernoulli polynomials of the second kind are defined by means of the following generating function

$$
\sum_{n=0}^{\infty} b_{n}(x) \frac{t^{n}}{n !}=\frac{t}{\log (1+t)}(1+t)^{x} .
$$

When $x=0, b_{n}(0):=b_{n}$ are called the Bernoulli numbers of the second kind, $c f .[16]$.

It is well-known from (1.7) that

$$
\left(\frac{t}{\log (1+t)}\right)^{r}(1+t)^{x-1}=\sum_{n=0}^{\infty} B_{n}^{(n-r+1)}(x) \frac{t^{n}}{n !},
$$

where $B_{n}^{(r)}(x)$ are the Bernoulli polynomials of order $r$, see [16]. 
The Stirling numbers of the first kind $S_{1}(n, k)$ and the Stirling numbers of the second kind $S_{2}(n, k)$ are defined (cf. $[2,4,5,12])$ by means of the following generating functions:

$$
\frac{(\log (1+t))^{k}}{k !}=\sum_{n=k}^{\infty} S_{1}(n, k) \frac{t^{n}}{n !} \text { and } \frac{\left(e^{t}-1\right)^{k}}{k !}=\sum_{n=k}^{\infty} S_{2}(n, k) \frac{t^{n}}{n !} .
$$

From (1.9), we get the following relations for $n \geq 0$ :

$$
(x)_{n}=\sum_{k=0}^{n} S_{1}(n, k) x^{k} \text { and } x^{n}=\sum_{k=0}^{n} S_{2}(n, k)(x)_{k} .
$$

Very recently, by means of the polyexponential function $\operatorname{Ei}_{k}(t), c f$. [13], defined by

$$
\operatorname{Ei}_{k}(t)=\sum_{n=1}^{\infty} \frac{t^{n}}{(n-1) ! n^{k}} \quad(k \in \mathbb{Z})
$$

as inverse to the polylogarithm function as follows:

$$
L i_{k}(t)=\sum_{n=1}^{\infty} \frac{t^{n}}{n^{k}} \quad(|t|<1 ; k \in \mathbb{Z}), c f .[6,8,10,24] .
$$

Kim-Kim [17] performed to generalize the degenerate Bernoulli polynomials as given below:

$$
\frac{\operatorname{Ei}_{k}(\log (1+t))}{e_{\lambda}(t)-1} e_{\lambda}^{x}(t)=\sum_{n=0}^{\infty} \beta_{n, \lambda}^{(k)}(x) \frac{t^{n}}{n !} .
$$

Upon setting $x=0$ in (1.13), $\beta_{n, \lambda}^{(k)}(0):=\beta_{n, \lambda}^{(k)}$ are called the degenerate poly-Bernoulli numbers.

For $k \in \mathbb{Z}$, the type 2 degenerate poly-Euler polynomials $\mathfrak{E}_{n, \lambda}^{(k)}(x)$ are defined, $c f$. [22], as follows:

$$
\frac{\operatorname{Ei}_{k}(\log (1+2 t))}{t\left(e_{\lambda}(t)+1\right)} e_{\lambda}^{x}(t)=\sum_{n=0}^{\infty} \mathfrak{E}_{n, \lambda}^{(k)}(x) \frac{t^{n}}{n !} .
$$

When $x=0, \mathfrak{E}_{n, \lambda}^{(k)}(0):=\mathfrak{E}_{n, \lambda}^{(k)}$ are called the type 2 degenerate poly-Euler numbers. Lee et al. [22] studied the type 2 degenerate poly-Euler polynomials and provided multifarious explicit formulas and identities.

Since $\operatorname{Ei}_{1}(t)=e^{t}-1$, it is worthy to note that

$$
\beta_{n, \lambda}^{(1)}(x):=B_{n, \lambda}(x) \text { and } \mathfrak{E}_{n, \lambda}^{(1)}(x):=E_{n, \lambda}(x) .
$$

In this paper, by means of the polyexponential function and degenerate exponential function, we introduce a class of new generating function for the Frobenius-Genocchi polynomials, called the type 2 degenerate polyFrobenius-Genocchi polynomials. Then, we investigate diverse formulas and identities covering some summation formulas, derivative formula, correlations with Bernoulli polynomials and numbers, Stirling numbers of the both kinds, degenerate Frobenius-Genocchi polynomials and degenerate Frobenius-Euler polynomials. Moreover, by using the unipoly function, we consider degenerate unipoly-Frobenius-Genocchi polynomials and derive some formulas and relationships with Daehee numbers, degenerate Frobenius-Genocchi numbers and Stirling numbers of the first kind. Finally, an Gaussian integral representation of the Frobenius-Genocchi polynomials is derived by means of the 2 -variable Hermite polynomials.

\section{The type 2 Degenerate Poly-Frobenius-Genocchi Polynomials}

Now, we consider the following Definition 1 by means of the polyexponential function.

Definition 1. Let $k \in \mathbb{Z}$. The type 2 degenerate poly-Frobenius-Genocchi polynomials are defined via the following exponential generating function (in a suitable neigbourhood of $t=0$ ) in terms of the polyexponential function as given below:

$$
\frac{\operatorname{Ei}_{k}(\log (1+(1-u) t))}{e_{\lambda}(t)-u} e_{\lambda}^{x}(t)=\sum_{n=0}^{\infty} G_{n, \lambda}^{(F, k)}(x ; u) \frac{t^{n}}{n !} .
$$


When $x=0$ in (2.1), $G_{n, \lambda}^{(F, k)}(0 ; u):=G_{n, \lambda}^{(F, k)}(u)$ is called type 2 degenerate poly-Frobenius-Genocchi numbers.

Remark 1. Taking $k=1$ in (2.1) yields $G_{n, \lambda}^{(F, 1)}(x ; u):=G_{n, \lambda}^{F}(x ; u)$ are the degenerate Frobenius-Genocchi polynomials $G_{n, \lambda}^{F}(x ; u)$ (cf. [9]) as follows:

$$
\frac{(1-u) t}{e_{\lambda}(t)-u} e_{\lambda}^{x}(t)=\sum_{n=0}^{\infty} G_{n, \lambda}^{F}(x ; u) \frac{t^{n}}{n !}
$$

Remark 2. Upon setting $\lambda \rightarrow 0$ in (2.1) gives $\lim _{\lambda \rightarrow 0} G_{n, \lambda}^{(F, k)}(x ; u):=G_{n}^{(F, k)}(x ; u)$ are type 2 poly-FrobeniusGenocchi polynomials $G_{n}^{(F, k)}(x ; u)(c f .[7])$ as follows:

$$
\frac{\operatorname{Ei}_{k}(\log (1+(1-u) t))}{e^{t}-u} e^{x t}=\sum_{n=0}^{\infty} G_{n}^{(F, k)}(x ; u) \frac{t^{n}}{n !} .
$$

Remark 3. Taking $k=1$ and $\lambda \rightarrow 0$ in (2.1) yields $G_{n, \lambda}^{(F, 1)}(x ;-1):=G_{n, \lambda}(x)$ are the Frobenius-Genocchi polynomials in (1.6).

Now, we give the following theorem.

Theorem 1. The following relation

$$
G_{n, \lambda}^{(F, k)}(x ; u)=\sum_{l=0}^{n}\left(\begin{array}{l}
n \\
l
\end{array}\right) G_{n-l, \lambda}^{(F, k)}(u)(x)_{l, \lambda}
$$

is valid for $k \in \mathbb{Z}$ and $n \geq 0$.

Proof. By Definition 1, we consider that

$$
\begin{aligned}
\sum_{n=0}^{\infty} G_{n, \lambda}^{(F, k)}(x ; u) \frac{t^{n}}{n !} & =\frac{\operatorname{Ei}_{k}(\log (1+(1-u) t))}{e_{\lambda}(t)-u} e_{\lambda}^{x}(t) \\
& =\left(\sum_{n=0}^{\infty} G_{n, \lambda}^{(F, k)}(u) \frac{t^{n}}{n !}\right)\left(\sum_{n=0}^{\infty}(x)_{n, \lambda} \frac{t^{n}}{n !}\right) \\
& =\sum_{n=0}^{\infty}\left(\sum_{l=0}^{n}\left(\begin{array}{c}
n \\
l
\end{array}\right) G_{n-l, \lambda}^{(F, k)}(u)(x)_{l, \lambda}\right) \frac{t^{n}}{n !}
\end{aligned}
$$

which implies the asserted result in (2.3).

Now, we give the partial derivative of type 2 degenerate Frobenius-Genocchi polynomials, with the respect to $x$, by the following theorem.

Theorem 2. The following relation

$$
\frac{\partial}{\partial x} G_{n, \lambda}^{(F, k)}(x ; u)=n ! \sum_{l=1}^{\infty} G_{n-l, \lambda}^{(F, k)}(x ; u) \frac{(-1)^{l+1}}{(n-l) ! l^{l-1}}
$$

is valid for $k \in \mathbb{Z}$ and $n \geq 0$. 
Proof. By Definition 1, we consider that

$$
\begin{aligned}
\sum_{n=0}^{\infty} \frac{\partial}{\partial x} G_{n, \lambda}^{(F, k)}(x ; u) \frac{t^{n}}{n !} & =\frac{\operatorname{Ei}_{k}(\log (1+(1-u) t))}{e_{\lambda}(t)-u} \frac{\partial}{\partial x} e_{\lambda}^{x}(t) \\
& =\left(\sum_{n=0}^{\infty} G_{n, \lambda}^{(F, k)}(x ; u) \frac{t^{n}}{n !}\right) \frac{1}{\lambda} \ln (1+\lambda t) \\
& =\left(\sum_{n=0}^{\infty} G_{n, \lambda}^{(F, k)}(x ; u) \frac{t^{n}}{n !}\right)\left(\sum_{l=1}^{\infty} \frac{(-1)^{l+1}}{l} \lambda^{l-1} t^{l}\right) \\
& =\sum_{n=0}^{\infty} \sum_{l=1}^{\infty} G_{n, \lambda}^{(F, k)}(x ; u) \frac{(-1)^{l+1}}{l} \lambda^{l-1} \frac{t^{n+l}}{n !},
\end{aligned}
$$

which implies the asserted result in (2.3).

A relation between the type 2 degenerate poly-Frobenius-Genocchi polynomials and the degenerate Frobenius-Genocchi polynomials is stated in the following theorem.

Theorem 3. For $k \in \mathbb{Z}$ and $n \geq 0$, we have

$$
G_{n, \lambda}^{(F, k)}(x ; u)=\sum_{m=0}^{n} \frac{(1-u)^{m}}{m+1} \sum_{l=1}^{m+1}\left(\begin{array}{c}
n \\
m
\end{array}\right) l^{1-k} S_{1}(m+1, l) G_{n-m, \lambda}^{F}(x ; u) .
$$

Proof. From (1.11), we observe that

$$
\begin{aligned}
\operatorname{Ei}_{k}(\log (1+(1-u) t)) & =\sum_{l=1}^{\infty} \frac{(\log (1+(1-u) t))^{l}}{(l-1) ! l^{k}} \\
& =\sum_{l=1}^{\infty} \frac{1}{l^{k-1}} \sum_{m=l}^{\infty} S_{1}(m, l)(1-u)^{m} \frac{t^{m}}{m !} \\
& =\sum_{m=0}^{\infty} \sum_{l=1}^{m+1} \frac{S_{1}(m+1, l)(1-u)^{m+1}}{l^{k-1}(m+1)} \frac{t^{m+1}}{m !} .
\end{aligned}
$$

Then, by (2.1), we get

$$
\begin{aligned}
\frac{t(1-u) e_{\lambda}^{x}(t)}{e_{\lambda}(t)-u} \frac{\operatorname{Ei}_{k}(\log (1+(1-u) t))}{t(1-u)} & =\left(\sum_{n=0}^{\infty} G_{n, \lambda}^{(F)}(x ; u) \frac{t^{n}}{n !}\right)\left(\sum_{m=0}^{\infty} \sum_{l=1}^{m+1} \frac{S_{1}(m+1, l)(1-u)^{m}}{l^{k-1}(m+1)} \frac{t^{m}}{m !}\right) \\
& =\sum_{n=0}^{\infty}\left(\sum_{m=0}^{n} \sum_{l=1}^{m+1}\left(\begin{array}{c}
n \\
m
\end{array}\right) \frac{S_{1}(m+1, l)(1-u)^{m}}{l^{k-1}(m+1)} G_{n-m, \lambda}^{F}(x ; u)\right) \frac{t^{n}}{n !},
\end{aligned}
$$

which means the asserted result in (2.5).

The immediate results of the Theorem 3 are stated below.

Corollary 1. Putting $x=0$ in Theorem 3 yields

$$
G_{n, \lambda}^{(F, k)}(u)=\sum_{m=0}^{n} \frac{(1-u)^{m}}{m+1} \sum_{l=1}^{m+1}\left(\begin{array}{c}
n \\
m
\end{array}\right) l^{1-k} S_{1}(m+1, l) G_{n-m, \lambda}^{F}(u) .
$$

Corollary 2. Taking $k=1$ in Theorem 3 gives

$$
G_{n, \lambda}^{(F, 1)}(x ; u)=\sum_{m=0}^{n} \frac{(1-u)^{m}}{m+1} \sum_{l=1}^{m+1}\left(\begin{array}{c}
n \\
m
\end{array}\right) S_{1}(m+1, l) G_{n-m, \lambda}^{F}(x ; u) .
$$


Corollary 3. Taking $k=1$ and $u=-1$ in Theorem 3 reduces

$$
G_{n, \lambda}^{F}(x)=\sum_{m=0}^{n} \frac{2^{m}}{m+1} \sum_{l=1}^{m+1}\left(\begin{array}{c}
n \\
m
\end{array}\right) S_{1}(m+1, l) G_{n-m, \lambda}(x) .
$$

The degenerate Frobenius-Euler polynomials $h_{n, \lambda}(x ; u)(c f$. [14]) are defined as follows:

$$
\frac{1-u}{e_{\lambda}(t)-u} e_{\lambda}^{x}(t)=\sum_{n=0}^{\infty} h_{n, \lambda}(x ; u) \frac{t^{n}}{n !}
$$

By (2.2) and (2.8), we have

$$
h_{n, \lambda}(x ; u)=\frac{G_{n+1, \lambda}^{F}(x ; u)}{n+1} .
$$

Then, by combining (2.2) and (2.8) with Theorem 3, we state the following Corollary.

Corollary 4. For $k \in \mathbb{Z}$ and $n \geq 0$, we have

$$
G_{n, \lambda}^{(F, k)}(x ; u)=\sum_{m=1}^{n} \sum_{l=1}^{m}\left(\begin{array}{c}
n \\
m
\end{array}\right) \frac{S_{1}(m, l)(1-u)^{m}}{l^{k-1}} h_{n-m, \lambda}(x ; u) .
$$

Here, we need the following lemma for Theorem 4.

Lemma 1. For $k \in \mathbb{Z}$ and $n \geq 0$, we have

$$
\frac{d}{d x} \operatorname{Ei}_{k}(\log (1+(1-u) x))=\frac{1-u}{(1+(1-u) x) \log (1+(1-u) x)} \operatorname{Ei}_{k-1}(\log (1+(1-u) x)) .
$$

Proof. From (1.11), we observe that

$$
\begin{aligned}
\frac{d}{d x} \operatorname{Ei}_{k}(\log (1+(1-u) x)) & =\frac{d}{d x} \sum_{l=1}^{\infty} \frac{(\log (1+(1-u) x))^{l}}{(l-1) ! l^{k}} \\
& =\frac{1-u}{(1+(1-u) x) \log (1+(1-u) x)} \sum_{l=1}^{\infty} \frac{(\log (1+(1-u) x))^{l}}{(l-1) ! l^{k-1}} \\
& =\frac{1-u}{(1+(1-u) x) \log (1+(1-u) x)} \operatorname{Ei}_{k-1}(\log (1+(1-u) x)),
\end{aligned}
$$

which is the claimed result in (2.10).

Theorem 4. Let $n$ be a nonnegative integer and $k \geq 2$. Then the following identity holds:

$$
\begin{aligned}
G_{n, \lambda}^{(F, k)}(u)= & \sum_{m=0}^{n}\left(\begin{array}{c}
n \\
m
\end{array}\right) \sum_{m_{1}+m_{2}+\cdots+m_{k-1}=m}^{\infty}\left(\begin{array}{c}
m \\
m_{1}, m_{2}, \cdots, m_{k-1}
\end{array}\right)(1-u)^{m_{1}+m_{2}+\cdots m_{k-1}} \\
& \times G_{n-m, \lambda}^{F}(u) \frac{B_{m_{1}}^{\left(m_{1}\right)}(0)}{m_{1}+1} \frac{B_{m_{2}}^{\left(m_{2}\right)}(0)}{m_{1}+m_{2}+1} \cdots \frac{B_{m_{k-1}}^{\left(m_{k-1}\right)}(0)}{m_{1}+m_{2}+\cdots+m_{k-1}+1}
\end{aligned}
$$

Proof. By (2.10), we consider

$$
\begin{gathered}
\operatorname{Ei}_{k}(\log (1+(1-u) x))=\int_{0}^{x} \frac{1-u}{(1+(1-u) x) \log (1+(1-u) x)} \operatorname{Ei}_{k-1}(\log (1+(1-u) x)) d t \\
=\int_{0}^{x} \frac{1-u}{(1+(1-u) x) \log (1+(1-u) x)} \\
\times \underbrace{\int_{0}^{t} \frac{1-u}{(1+(1-u) x) \log (1+(1-u) x)} \cdots \int_{0}^{t} \frac{(1-u)^{2} t}{(1+(1-u) x) \log (1+(1-u) x)}}_{(k-2) \text { times }} d t d t \cdots d t .
\end{gathered}
$$


Then, we obtain

$$
\begin{aligned}
& \sum_{n=0}^{\infty} G_{n, \lambda}^{(F, k)}(u) \frac{t^{n}}{n !}=\frac{\operatorname{Ei}_{k}(\log (1+(1-u) t))}{e_{\lambda}(t)-u} \\
& =\frac{1}{e_{\lambda}(t)-u} \int_{0}^{x} \frac{1-u}{(1+(1-u) x) \log (1+(1-u) x)} \\
& \times \underbrace{\int_{0}^{t} \frac{1-u}{(1+(1-u) x) \log (1+(1-u) x)} \cdots \int_{0}^{t} \frac{(1-u)^{2} t}{(1+(1-u) x) \log (1+(1-u) x)}}_{(k-2) \text { times }} d t d t \cdots d t \\
& =\frac{(1-u) x}{e_{\lambda}(t)-u} \sum_{m=0}^{\infty} \sum_{m_{1}+m_{2}+\cdots+m_{k-1}=m}^{\infty}\left(\begin{array}{c}
m \\
m_{1}, m_{2}, \cdots, m_{k-1}
\end{array}\right)(1-u)^{m_{1}+m_{2}+\cdots m_{k-1}} \\
& \times \frac{B_{m_{1}}^{\left(m_{1}\right)}(0)}{m_{1}+1} \frac{B_{m_{2}}^{\left(m_{2}\right)}(0)}{m_{1}+m_{2}+1} \cdots \frac{B_{m_{k-1}}^{\left(m_{k-1}\right)}(0)}{m_{1}+m_{2}+\cdots+m_{k-1}+1} \frac{x^{m}}{m !} \\
& =\sum_{n=0}^{\infty} \sum_{m=0}^{n}\left(\begin{array}{l}
n \\
m
\end{array}\right) \sum_{m_{1}+m_{2}+\cdots+m_{k-1}=m}^{\infty}\left(\begin{array}{c}
m \\
m_{1}, m_{2}, \cdots, m_{k-1}
\end{array}\right)(1-u)^{m_{1}+m_{2}+\cdots m_{k-1}} \\
& \times G_{n-m, \lambda}^{F}(u) \frac{B_{m_{1}}^{\left(m_{1}\right)}(0)}{m_{1}+1} \frac{B_{m_{2}}^{\left(m_{2}\right)}(0)}{m_{1}+m_{2}+1} \cdots \frac{B_{m_{k-1}}^{\left(m_{k-1}\right)}(0)}{m_{1}+m_{2}+\cdots+m_{k-1}+1} \frac{x^{n}}{n !} .
\end{aligned}
$$

This finalizes the proof of the theorem.

Now, we give the following theorem.

Theorem 5. For $k \in \mathbb{Z}$ and $n \in \mathbb{N}_{0}$, we have

$$
\sum_{m=0}^{n} \frac{S_{2}(n, m)}{(1-u)^{m}} G_{m, \lambda}^{(F, k)}(u)=\sum_{m=0}^{n} \sum_{i=0}^{m} \sum_{j=0}^{i}\left(\begin{array}{c}
n \\
m
\end{array}\right)\left(\begin{array}{c}
m \\
i
\end{array}\right) \frac{G_{j, \lambda}^{(F)}(u) S_{2}(i, j)}{(1-u)^{j}} \frac{B_{m-i}}{(n-m+1)^{k}}
$$

Proof. Replacing $t$ by $\frac{e^{t}-1}{1-u}$ in (2.1), we attain

$$
\begin{aligned}
\frac{\operatorname{Ei}_{k}(t)}{e_{\lambda}\left(\frac{e^{t}-1}{1-u}\right)-u} & =\sum_{m=0}^{\infty}(1-u)^{-m} G_{m, \lambda}^{(F, k)}(u) \frac{\left(e^{t}-1\right)^{m}}{m !} \\
& =\sum_{m=0}^{\infty}(1-u)^{-m} G_{m, \lambda}^{(F, k)}(u) \sum_{n=0}^{\infty} S_{2}(n, m) \frac{t^{n}}{n !} \\
& =\sum_{n=0}^{\infty} \sum_{m=0}^{n} \frac{S_{2}(n, m)}{(1-u)^{m}} G_{m, \lambda}^{(F, k)}(u) \frac{t^{n}}{n !}
\end{aligned}
$$


Also, we investigate

$$
\begin{gathered}
\frac{(1-u)\left(\frac{e^{t}-1}{1-u}\right)}{e_{\lambda}\left(\frac{e^{t}-1}{1-u}\right)-u} \frac{1}{e^{t}-1} \sum_{l=1}^{\infty} \frac{t^{l}}{(l-1) ! l^{k}}=\frac{(1-u)\left(\frac{e^{t}-1}{1-u}\right)}{e_{\lambda}\left(\frac{e^{t}-1}{1-u}\right)-u} \frac{t}{e^{t}-1} \sum_{l=0}^{\infty} \frac{t^{l}}{l !(l+1)^{k}} \\
=\sum_{j=0}^{\infty}(1-u)^{-j} G_{j, \lambda}^{(F)}(u) \frac{\left(e^{t}-1\right)^{j}}{j !} \sum_{m=0}^{\infty} B_{m} \frac{t^{m}}{m !} \sum_{l=0}^{\infty} \frac{t^{l}}{l !(l+1)^{k}} \\
=\sum_{i=0}^{\infty} \sum_{j=0}^{i}(1-u)^{-j} G_{j, \lambda}^{(F)}(u) S_{2}(i, j) \frac{t^{i}}{i !} \sum_{m=0}^{\infty} B_{m} \frac{t^{m}}{m !} \sum_{l=0}^{\infty} \frac{t^{l}}{l !(l+1)^{k}} \\
=\sum_{m=0}^{\infty} \sum_{i=0}^{m} \sum_{j=0}^{i}\left(\begin{array}{c}
m \\
i
\end{array}\right) \frac{G_{j, \lambda}^{(F)}(u) S_{2}(i, j)}{(1-u)^{j}} B_{m-i} \frac{t^{m}}{m !} \sum_{l=0}^{\infty} \frac{t^{l}}{l !(l+1)^{k}} \\
=\sum_{n=0}^{\infty} \sum_{m=0}^{n} \sum_{i=0}^{m} \sum_{j=0}^{i}\left(\begin{array}{c}
n \\
m
\end{array}\right)\left(\begin{array}{c}
m \\
i
\end{array}\right) \frac{G_{j, \lambda}^{(F)}(u) S_{2}(i, j)}{(1-u)^{j}} \frac{B_{m-i}}{(n-m+1)^{k}} \frac{t^{n}}{n !} .
\end{gathered}
$$

This completes proof of the theorem.

Theorem 6. For $k \in \mathbb{Z}$ and $n \geq 0$, we have

$$
G_{n, \lambda}^{(F, k)}(x+1 ; u)-u G_{n, \lambda}^{(F, k)}(x ; u)=\sum_{m=0}^{n} \sum_{l=1}^{m+1}\left(\begin{array}{c}
n \\
m
\end{array}\right) \frac{S_{1}(m+1, l)(1-u)^{m+1}}{l^{k-1}(m+1)}(x)_{n-m, \lambda} .
$$

Proof. By Definition 1 and formula (2.6), we see that

$$
\begin{aligned}
\sum_{n=0}^{\infty}\left(G_{n, \lambda}^{(F, k)}(x+1 ; u)-u G_{n, \lambda}^{(F, k)}(x ; u)\right) \frac{t^{n}}{n !} & =\operatorname{Ei}_{k}(\log (1+(1-u) t)) e_{\lambda}^{x}(t) \\
& =\left(\sum_{m=0}^{\infty} \sum_{l=1}^{m+1} \frac{S_{1}(m+1, l)(1-u)^{m+1}}{l^{k-1}(m+1)} \frac{t^{m+1}}{m !}\right)\left(\sum_{n=0}^{\infty}(x)_{n, \lambda} \frac{t^{n}}{n !}\right) \\
& =\sum_{n=0}^{\infty}\left(\sum_{m=0}^{n} \sum_{l=1}^{m+1}\left(\begin{array}{c}
n \\
m
\end{array}\right) \frac{S_{1}(m+1, l)(1-u)^{m+1}}{l^{k-1}(m+1)}(x)_{n-m, \lambda}\right) \frac{t^{n}}{n !},
\end{aligned}
$$

which gives the asserted result in (2.11).

\section{On the degenerate Unipoly-Frobenius-Genocchi Polynomials}

In this section, Kim-Kim [13] introduced unipoly function $u_{k}(x \mid p)$ attached to $p$ being any arithmetic function that is a real or complex valued function defined on the set of positive integers as follows:

$$
u_{k}(x \mid p)=\sum_{n=1}^{\infty} \frac{p(n)}{n^{k}} x^{n}, \quad(k \in \mathbb{Z}) .
$$

It is readily seen that

$$
u_{k}(x \mid 1)=\sum_{n=1}^{\infty} \frac{x^{n}}{n^{k}}=L i_{k}(x)
$$

is the polylogarithm function given in (1.12). By means of the unipoly function, Kim-Kim [13] defined unipoly-Bernoulli polynomials as follows:

$$
\sum_{n=0}^{\infty} B_{n, p}^{(k)}(x) \frac{t^{n}}{n !}=\frac{u_{k}\left(1-e^{-t} \mid p\right)}{1-e^{-t}} e^{x t} .
$$

They provide several formulae and relations for these polynomials, see [13]. 
With this motivation, by means of the unipoly function attached to $p$ given in (3.1), we introduce degenerate unipoly-Frobenius-Genocchi polynomials by means of the following exponential generating function:

$$
\sum_{n=0}^{\infty} G_{n, p ; \lambda}^{(F, k)}(x ; u) \frac{t^{n}}{n !}=\frac{u_{k}(\log (1+(1-u) t) \mid p)}{e_{\lambda}(t)-u} e_{\lambda}^{x}(t) .
$$

Note that taking $x=0$ in $(3.2), G_{n, p ; \lambda}^{(F, k)}(0 ; u):=G_{n, p ; \lambda}^{(F, k)}(u)$ are called the degenerate unipoly-FrobeniusGenocchi numbers.

By (3.2), we consider that

$$
\begin{aligned}
\sum_{n=0}^{\infty} G_{n, p ; \lambda}^{(F, k)}(x ; u) \frac{t^{n}}{n !} & =\frac{u_{k}(\log (1+(1-u) t) \mid p)}{e_{\lambda}(t)-u} e_{\lambda}^{x}(t) \\
& =\left(\sum_{n=0}^{\infty} G_{n, p ; \lambda}^{(F, k)}(u) \frac{t^{n}}{n !}\right)\left(\sum_{n=0}^{\infty}(x)_{n, \lambda} \frac{t^{n}}{n !}\right) \\
& =\sum_{n=0}^{\infty}\left(\sum_{l=0}^{n}\left(\begin{array}{l}
n \\
l
\end{array}\right) G_{n-l, p ; \lambda}^{(F, k)}(u)(x)_{l, \lambda}\right) \frac{t^{n}}{n !}
\end{aligned}
$$

Hence, we give the following theorem.

Theorem 7. The following relation

$$
G_{n, p ; \lambda}^{(F, k)}(x ; u)=\sum_{l=0}^{n}\left(\begin{array}{c}
n \\
l
\end{array}\right) G_{n-l, p ; \lambda}^{(F, k)}(u)(x)_{l, \lambda}
$$

is ture for $k \in \mathbb{Z}$ and $n \geq 0$.

We observe that

$$
\begin{aligned}
\sum_{n=0}^{\infty} \frac{\partial}{\partial x} G_{n, p ; \lambda}^{(F, k)}(x ; u) \frac{t^{n}}{n !} & =\frac{u_{k}(\log (1+(1-u) t) \mid p)}{e_{\lambda}(t)-u} \frac{\partial}{\partial x} e_{\lambda}^{x}(t) \\
& =\left(\sum_{n=0}^{\infty} G_{n, p ; \lambda}^{(F, k)}(x ; u) \frac{t^{n}}{n !}\right)\left(\frac{1}{\lambda} \ln (1+\lambda t)\right) \\
& =\left(\sum_{n=0}^{\infty} G_{n, p ; \lambda}^{(F, k)}(x ; u) \frac{t^{n}}{n !}\right)\left(\sum_{l=1}^{\infty} \frac{(-1)^{l+1}}{l} \lambda^{l-1} t^{l}\right) \\
& =\sum_{n=0}^{\infty} \sum_{l=1}^{\infty} G_{n, p ; \lambda}^{(F, k)}(x ; u) \frac{(-1)^{l+1}}{l} \lambda^{l-1} \frac{t^{n+l}}{n !},
\end{aligned}
$$

Therefore, we give the partial derivative, with respect to $x$, for the degenerate unipoly-Frobenius-Genocchi polynomials by following theorem.

Theorem 8. Let $k \in \mathbb{Z}$ and $n \geq 0$. We have the following derivative rule:

$$
\frac{\partial}{\partial x} G_{n, p ; \lambda}^{(F, k)}(x ; u)=n ! \sum_{l=1}^{\infty} G_{n-l, p ; \lambda}^{(F, k)}(x ; u) \frac{(-1)^{l+1}}{(n-l) ! l} \lambda^{l-1} .
$$


Upon setting $p(n)=\frac{1}{\Gamma(n)}$ in $(3.2)$, we acquire

$$
\begin{aligned}
\sum_{n=0}^{\infty} G_{n, p ; \lambda}^{(F, k)}(u) \frac{t^{n}}{n !} & =\frac{1}{e_{\lambda}(t)-u} u_{k}\left(\log (1+(1-u) t) \mid \frac{1}{\Gamma}\right) \\
& =\frac{1}{e_{\lambda}(t)-u} \sum_{m=1}^{\infty} \frac{(\log (1+(1-u) t))^{m}}{m^{k}(m-1) !} \\
& =\frac{1}{e_{\lambda}(t)-u} e_{k}(\log (1+(1-u) t)) \\
& =\sum_{n=0}^{\infty} G_{n ; \lambda}^{(F, k)}(u) \frac{t^{n}}{n !},
\end{aligned}
$$

which gives the following relation:

$$
G_{n, \frac{1}{\Gamma} ; \lambda}^{(F, k)}(u)=G_{n ; \lambda}^{(F, k)}(u) .
$$

From (3.4), we have

$$
\begin{aligned}
\sum_{n=0}^{\infty} G_{n, p ; \lambda}^{(F, k)}(u) \frac{t^{n}}{n !} & =\frac{1}{e_{\lambda}(t)-u} \sum_{m=1}^{\infty} \frac{p(m)}{m^{k}}(\log (1+(1-u) t))^{m} \\
& =\frac{1}{e_{\lambda}(t)-u} \sum_{m=0}^{\infty} \frac{p(m+1)(m+1) !}{(m+1)^{k}} \frac{(\log (1+(1-u) t))^{m+1}}{(m+1) !} \\
& =\frac{1}{e_{\lambda}(t)-u} \sum_{m=0}^{\infty} \frac{p(m+1)(m+1) !}{(m+1)^{k}} \sum_{n=m+1}^{\infty} S_{1}(n, m+1)(1-u)^{n} \frac{t^{n}}{n !} \\
& =\frac{(1-u) t}{e_{\lambda}(t)-u} \sum_{m=0}^{\infty} \frac{p(m+1)(m+1) !}{(m+1)^{k}} \sum_{n=m}^{\infty} S_{1}(n+1, m+1)(1-u)^{n} \frac{t^{n}}{(n+1) !} \\
& =\sum_{n=0}^{\infty} G_{n ; \lambda}^{F}(u) \frac{t^{n}}{n !} \sum_{n=0}^{\infty}\left(\sum_{m=0}^{n} \frac{p(m+1)(m+1) !}{(m+1)^{k}} \frac{S_{1}(n+1, m+1)}{n+1}(1-u)^{n}\right) \frac{t^{n}}{n !} \\
& =\sum_{n=0}^{\infty}\left(\sum_{l=0}^{n} \sum_{m=0}^{l}\left(\begin{array}{l}
n \\
l
\end{array}\right) \frac{p(m+1)(m+1) !}{(m+1)^{k}} \frac{S_{1}(l+1, m+1)}{l+1}(1-u)^{l} G_{n-l ; \lambda}^{F}(u)\right) \frac{t^{n}}{n !},
\end{aligned}
$$

which yields the following theorem.

Theorem 9. For $k \in \mathbb{Z}$ and $n \geq 0$, we have

$$
G_{n, p ; \lambda}^{(F, k)}(u)=\sum_{l=0}^{n} \sum_{m=0}^{l}\left(\begin{array}{l}
n \\
l
\end{array}\right) \frac{p(m+1)(m+1) !}{(m+1)^{k}} \frac{S_{1}(l+1, m+1)}{l+1}(1-u)^{l} G_{n-l ; \lambda}^{F}(u) .
$$

Particularly, for $p(n)=\frac{1}{\Gamma(n)}$, we get

$$
G_{n, \frac{1}{\Gamma} ; \lambda}^{(F, k)}(u)=\sum_{l=0}^{n} \sum_{m=0}^{l}\left(\begin{array}{l}
n \\
l
\end{array}\right) \frac{m+1}{(m+1)^{k}} \frac{S_{1}(l+1, m+1)}{l+1}(1-u)^{l} G_{n-l ; \lambda}^{F}(u) .
$$


Now, we consider

$$
\begin{aligned}
\sum_{n=0}^{\infty} G_{n, p ; \lambda}^{(F, k)}(u) \frac{t^{n}}{n !}= & \frac{1}{e_{\lambda}(t)-u} \sum_{m=1}^{\infty} \frac{p(m)}{m^{k}}(\log (1+(1-u) t))^{m} \\
= & \frac{t(1-u)}{e_{\lambda}(t)-u} \frac{\log (1+(1-u) t)}{t(1-u)} \sum_{m=0}^{\infty} \frac{p(m+1) m !}{(m+1)^{k}} \frac{(\log (1+(1-u) t))^{m}}{m !} \\
= & \sum_{n=0}^{\infty} G_{n ; \lambda}^{F}(u) \frac{t^{n}}{n !} \sum_{n=0}^{\infty} D_{n} \frac{(1-u)^{n} t^{n}}{n !} \sum_{m=0}^{\infty} \frac{p(m+1) m !}{(m+1)^{k}} \sum_{n=m}^{\infty} S_{1}(n, m)(1-u)^{n} \frac{t^{n}}{n !} \\
= & \sum_{n=0}^{\infty}\left(\sum_{j=0}^{n}\left(\begin{array}{c}
n \\
j
\end{array}\right) G_{n-j ; \lambda}^{F}(u) D_{j}(1-u)^{j}\right) \frac{t^{n}}{n !} \\
& \times \sum_{n=0}^{\infty}\left(\sum_{m=0}^{n} \frac{p(m+1) m !}{(m+1)^{k}} S_{1}(n, m)(1-u)^{n}\right) \frac{t^{n}}{n !} \\
= & \sum_{n=0}^{\infty} \sum_{l=0}^{n} \sum_{j=0}^{n-l} \sum_{m=0}^{l}\left(\begin{array}{c}
n \\
l
\end{array}\right)\left(\begin{array}{c}
n-l \\
j
\end{array}\right) G_{n-j-l ; \lambda}^{F}(u) D_{j} \frac{p(m+1) m !}{(m+1)^{k}} S_{1}(l, m)(1-u)^{l+j} \frac{t^{n}}{n !} .
\end{aligned}
$$

where $D_{n}$ are the Daehee numbers defined by (cf. [12])

$$
\sum_{n=0}^{\infty} D_{n} \frac{t^{n}}{n !}=\frac{\log (1+t)}{t}, \text { see }[12] .
$$

Thus, we give the following theorem.

Theorem 10. For $k \in \mathbb{Z}$ and $n \geq 0$, we have

$$
G_{n, p ; \lambda}^{(F, k)}(u)=\sum_{l=0}^{n} \sum_{j=0}^{n-l} \sum_{m=0}^{l}\left(\begin{array}{c}
n \\
l
\end{array}\right)\left(\begin{array}{c}
n-l \\
j
\end{array}\right) G_{n-j-l ; \lambda}^{F}(u) D_{j} \frac{p(m+1) m !}{(m+1)^{k}} S_{1}(l, m)(1-u)^{l+j} .
$$

\section{Concluding Remarks}

Gaussian integral representation plays an important role in classical problems arising from quantum optics and quantum mechanics. They are exploited to calculate the optical mode overlapping and transition rates between quantum eigenstates of the harmonic oscillator, cf. [1].

Let us now consider the following integral:

$$
S_{n}(a, b, c ; u):=S_{n}:=\int_{-\infty}^{\infty} G_{n}^{F}(a x ; u) e^{-b x^{2}+c x} d x
$$

By (4.1), we have

By (1.6), we see that

$$
\sum_{n=0}^{\infty} S_{n} \frac{t^{n}}{n !}=\sum_{n=0}^{\infty}\left(\int_{-\infty}^{\infty} G_{n}^{F}(a x ; u) e^{-b x^{2}+c x} d x\right) \frac{t^{n}}{n !}
$$

Since

$$
\sum_{n=0}^{\infty} S_{n} \frac{t^{n}}{n !}=\frac{(1-u) t}{e^{t}-u} \int_{-\infty}^{\infty} e^{(a t+c) x-b x^{2}} d x
$$

which represents Gaussian integral, we find

$$
\int_{-\infty}^{\infty} e^{b x-a x^{2}+c} d x=\frac{\sqrt{\pi}}{\sqrt{a}} e^{\frac{b^{2}}{4 a}+c},
$$

$$
\sum_{n=0}^{\infty} S_{n} \frac{t^{n}}{n !}=\frac{\sqrt{\pi}}{\sqrt{b}} \frac{(1-u) t}{e^{t}-u} \exp \left(\frac{a^{2}}{4 b} t^{2}+\frac{c^{2}}{4 b}+\frac{a c}{2 b} t\right)
$$


with the assumption $\exp (t):=e^{t}$. Recall from [2] that the 2-variable Hermite polynomials are defined by means of the following generating function:

$$
\sum_{n=0}^{\infty} H_{n}(x, y) \frac{t^{n}}{n !}=\exp \left(x t+y t^{2}\right) .
$$

By (1.6) and (4.2), we derive

$$
\begin{aligned}
\sum_{n=0}^{\infty} S_{n} \frac{t^{n}}{n !} & =\frac{\sqrt{\pi}}{\sqrt{b}} e^{\frac{c^{2}}{4 b}}\left(\sum_{n=0}^{\infty} G_{n}^{F}(u) \frac{t^{n}}{n !}\right)\left(\sum_{n=0}^{\infty} H_{n}\left(\frac{a c}{2 b}, \frac{a^{2}}{4 b}\right) \frac{t^{n}}{n !}\right) \\
& =\frac{\sqrt{\pi}}{\sqrt{b}} e^{\frac{c^{2}}{4 b}} \sum_{n=0}^{\infty}\left(\sum_{k=0}^{n}\left(\begin{array}{l}
n \\
k
\end{array}\right) G_{n-k}^{F}(u) H_{k}\left(\frac{a c}{2 b}, \frac{a^{2}}{4 b}\right)\right) \frac{t^{n}}{n !} .
\end{aligned}
$$

By comparing the coefficients of $\frac{t^{n}}{n !}$ on the above, we obtain

$$
S_{n}=\frac{\sqrt{\pi}}{\sqrt{b}} e^{\frac{c^{2}}{4 b}} \sum_{k=0}^{n}\left(\begin{array}{l}
n \\
k
\end{array}\right) G_{n-k}^{F}(u) H_{k}\left(\frac{a c}{2 b}, \frac{a^{2}}{4 b}\right) .
$$

Thus, by (4.1) and (4.3), we finalize our paper with the following result:

$$
\int_{-\infty}^{\infty} G_{n}^{F}(a x ; u) e^{-b x^{2}+c x} d x=\frac{\sqrt{\pi}}{\sqrt{b}} e^{\frac{c^{2}}{4 b}} \sum_{k=0}^{n}\left(\begin{array}{l}
n \\
k
\end{array}\right) G_{n-k}^{F}(u) H_{k}\left(\frac{a c}{2 b}, \frac{a^{2}}{4 b}\right) .
$$

\section{References}

[1] Andrews, L. C. Special functions for applied mathematics and engineering. MacMillan, New York,1985.

[2] Appell, P.; Hermitt Kampé de Fériet, J. Fonctions hypergéomtriques et hypersphériques: polynomes d Hermite; GauthierVillars: Paris, France, 1926.

[3] Araci, S., Acikgoz, M. Construction of Fourier expansion of Apostol Frobenius-Euler polynomials and its applications. Adv. Differ. Equ. 2018, 67 (2018).

[4] Araci, S. Novel identities involving Genocchi numbers and polynomials arising from applications of umbral calculus. Appl. Math. Comput. 233 (2014), 599-607.

[5] Cangul, I.N.; Kurt, V.; Ozden, H.; Simsek, Y. On the higher-order $w$-q-Genocchi numbers. Adv. Stud. Contemp. Math. (Kyungshang) 2009, 19, 39-57.

[6] Dolgy, D.V., Jang, L.C. Some identities on the poly-Genocchi polynomials and numbers. Symmetry, 2020, $12,1007$.

[7] Duran, U., Acikgoz, M., Araci, Construction of the type 2 poly-Frobenius-Genocchi polynomials with their certain applications, Adv. Difference Equ., 2020, 432 (2020).

[8] Eastham, M. S. P. On polylogarithms. Proc. Glasgow Math. Assoc. 6, 1964, 169-171.

[9] Jang, L.-C., Lee, J. G., Rim, S.-H., Park. S., On the degenerate Frobenius-Genocchi polynomials, Glob. J. Pure Appl. Math., 2015, 11(5), 3601-3613.

[10] Khan, N., Usman, T., Nisar, K.S. A study of generalized Laguerre poly-Genocchi polynomials. Mathematics $2019,7,219$.

[11] Khan, W. A., Srivastava, D. On the generalized Apostol-type Frobenius-Genocchi polynomials. Filomat, 33:7 (2019), 1967-1977.

[12] Kim, D. S., Kim, T. Daehee numbers and polynomials, Appl. Math. Sci., Vol. 7, 2013, no. 120, 5969-5976.

[13] Kim, D.-S., Kim, T. A note on polyexponential and unipoly functions, Russ. J. Math. Phys, 26(1), 2019, 40-49.

[14] Kim, T., Kwon, H.-I., Seo, J.-J. On the degenerate Frobenius-Euler polynomials, Glob. J. Pure Appl. Math. 11 (2015), 2077-2084.

[15] Kim, T., Kim, D.S., Kwon, J.K., Kim, H.Y. A note on degenerate Genocchi and poly-Genocchi numbers and polynomials. J. Inequal. Appl. 2020, 2020, 110

[16] Kim, T., Kim, D.S., Kwon, J.K., Lee, H.S. Degenerate polyexponential functions and type 2 degenerate poly-Bernoulli numbers and polynomials. Adv. Difference Equ. 2020, 2020, 168

[17] Kim, T., Rim, S. H., Kim, H.Y., Jang, L.C. Degenerate poly-Bernoulli numbers and polynomials, Informatica, 2020, 31(3), 2-8.

[18] Kim, T. Some identities for the Bernoulli, the Euler and the Genocchi numbers and polynomials. Adv. Stud. Contemp. Math. 2010, 20, 23-28.

[19] Kim T., Jang, L.-C., Kim, D. S., Kim, H. Y., Some identities on type 2 degenerate Bernoulli polynomials of the second kind, Symmetry, 2020, 12(4), 510 .

[20] Kim, T., Khan, W. A., Sharma, S. K., Ghayasuddin, M., A note on parametric kinds of the degenerate poly-Bernoulli and poly-Genocchi polynomials, Symmetry, 2020, 12(4), 614 . 
[21] Kumam, W., Srivastava, H.M., Wani, S.A., Araci, S., Kumam, P. Truncated-exponential-based Frobenius-Euler polynomials. Adv. Difference Equ. 2019, 530 (2019).

[22] Lee, D.S., Kim, H.K., Jang, L.-C. Type 2 degenerate poly-Euler polynomials, Symmetry, 2020, $12,1011$.

[23] Lim, D., Some identities of degenerate Genocchi polynomials, Bull. Korean Math. Soc., 2016, 53(2), 569-579.

[24] Qin, S. Fully Degenerate poly-Genocchi polynomials, Pure Mathematics, 2020, 10(4), 345-355.

[25] Srivastava, H.M., Kurt, B., Simsek, Y. Some families of Genocchi type polynomials and their interpolation functions. Integral Transforms Spec. Funct. 2012, 23, 919-938.

[26] Wani, S. A., Khan, S., Nahid, T. Gould-Hopper based Frobenius-Genocchi polynomials and their generalized form, Afrika Mat., 2020, doi: 10.1007/s13370-020-00804-2.

[27] Yasar, B.Y., Ozarslan, M. A. Frobenius-Euler and Frobenius-Genocchi polynomials and their differential equations, New Trends Math. Sci., 3(2) (2015) 172-180. 